\title{
Improved Analysis of ECHO-256
}

\author{
Jérémy Jean ${ }^{1}$, María Naya-Plasencia ${ }^{2}$, and Martin Schläffer ${ }^{3}$ \\ 1 Ecole Normale Supérieure, France \\ 2 FHNW, Windisch, Switzerland \\ 3 IAIK, Graz University of Technology, Austria
}

\begin{abstract}
ECHO-256 is a second-round candidate of the SHA-3 competition. It is an AES-based hash function that has attracted a lot of interest and analysis. Up to now, the best known attacks were a distinguisher on the full internal permutation and a collision on four rounds of its compression function. The latter was the best known analysis on the compression function as well as the one on the largest number of rounds so far. In this paper, we extend the compression function results to get a distinguisher on 7 out of 8 rounds using rebound techniques. We also present the first 5-round collision attack on the ECHO-256 hash function.
\end{abstract}

Keywords: hash function, cryptanalysis, rebound attack, collision attack, distinguisher.

\section{Introduction}

ECHO-256 [1] is the 256-bit version of one of the second-round candidates of the SHA-3 competition. It is an AES-based hash function that has been the subject of many studies. Currently, the best known analysis of ECHO-256 are a distinguisher on the full 8-round internal permutation proposed in [13] and improved in [10]. Furthermore, a 4-round collision attack of the compression function has been presented in [4]. A previous analysis due to Schläffer in [14] has been shown to be incorrect in [4], but it introduced an alternative description of the ECHO round-function, which has then been reused in several analyses, including this paper. The best results of this paper are a collision attack on the hash function reduced to 5 rounds and a distinguisher of the compression function on 7 rounds. Additionally, we cover two more attacks in the Appendix. The complexities of previous results and our proposed attacks are reported in Table 1 .

Apart from the improved attacks on ECHO-256, this paper also covers a number of new techniques. The merging process of multiple inbound phases has been improved to find solutions also for the hash function, where much less freedom is available in the chaining input. For the hash function collision attack on 5

\footnotetext{
* This work was supported in part by ANR/SAPHIR II, by the French DGA, by the NCCR-MICS under grant number 5005-67322, by the ECRYPT II contract ICT2007-216646, by the Austrian FWF project P21936 and by the IAP Programme P6/26 BCRYPT of the Belgian State.
} 
Table 1. Best known cryptanalysis results on ECHO-256

\begin{tabular}{|c|c|c|c|c|c|}
\hline Rounds & Time & Memory & Generic & Type & Reference \\
\hline 8 & $2^{182}$ & $2^{37}$ & $2^{256}$ & Internal Permutation Distinguisher & {$[13]$} \\
\hline 8 & $2^{151}$ & $2^{67}$ & $2^{256}$ & Internal Permutation Distinguisher & {$[10]$} \\
\hline 4 & $2^{52}$ & $2^{16}$ & $2^{256}$ & Compression Function Collision & {$[4]$} \\
\hline 5 & $2^{112}$ & $2^{85.3}$ & $2^{128}$ & Hash Function Collision & Section 3 \\
\hline 6 & $2^{193}$ & $2^{128}$ & $2^{256}$ & Compression Function Collision & Section 4 \\
\hline 7 & $2^{193}$ & $2^{128}$ & $2^{240}$ & Compression Function Distinguisher & Section 4 \\
\hline
\end{tabular}

rounds, we use subspace differences which collide with a high probability at the output of the hash function. Additionally, we use multiple phases also in the outbound part to reduce the overall complexity of the attacks. For the 7 -round compression function distinguisher, we use the new techniques and algorithms introduced in [10, 11].

Outline. The paper is organized as follows. In Section 2 , we describe the 256bit version of the ECHO hash function and detail an alternative view that has already been used in several analysis [4, 14]. In particular, we emphasize the SuperMixColumns and SuperSBox transformations that ease the analysis. In Section 3, we provide a collision attack on this hash function reduced to 5 rounds and a distinguisher of the 7-round compression function in Section 4 .

In the extended version of this paper [5], we describe a chosen-salt collision attack on the 6-round compression function and a chosen-salt distinguisher for the compression function reduced to 7 rounds. We also improve the result from [4] into a collision attack on the 4-round ECHO-256 hash function.

\section{2 ЕСНО-256 Description}

$\mathrm{ECHO}$ is an iterated hash function and the compression function of ECHO updates an internal state described by a $16 \times 16$ matrix of GF $\left(2^{8}\right)$ elements, which can also be viewed as a $4 \times 4$ matrix of 16 AES states. Transformations on this large 2048-bit state are very similar to the one of the AES, the main difference being the equivalent S-Box called BigSubWords, which consists in two AES rounds. The diffusion of the AES states in ECHO is ensured by two big transformations: BigShiftRows and BigMixColumns (Figure 1).

At the end of the permutation, the BigFinal operation adds the current state to the initial one (feed-forward) and, in the case of ECHO-256, adds its four columns together to produce the new chaining value. In this paper, we only focus on ECHO256 and refer to the original publication [1] for more details on both ECHO-256 and ECHO-512 versions. Note that the keys used in the two AES rounds are an internal counter and the salt, respectively: they are mainly introduced to break the existing symmetries of the AES unkeyed permutation [7]. Since we are not 


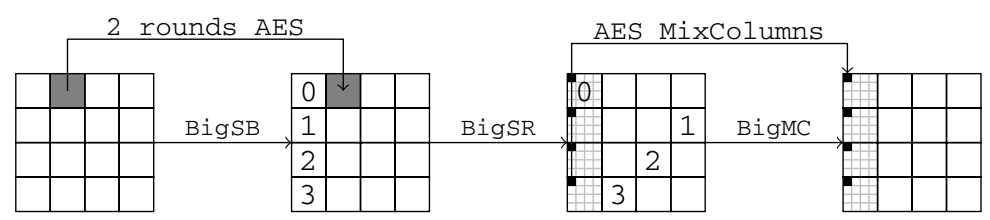

Fig. 1. One round of the ECHO permutation. Each of the 16 cells is an AES state.

using any property relying on symmetry and adding constants does not change differences, we omit these steps in the following.

Two versions of the hash function ECHO have been submitted to the SHA-3 contest: ECHO-256 and ECHO-512, which share the same state size and round function, but inject messages of size 1536 or 1024 bits respectively in the compression function. Note that the message is padded by adding a single 1 followed by zeros to fill up the last message block. The last 18 bytes of the last message block always contain the 2-byte hash output size, followed by the 16-byte message length. Focusing on ECHO-256 and denoting $f$ its compression function, $H_{i}$ the $i$-th output chaining value, $M_{i}=M_{i}^{0}\left\|M_{i}^{1}\right\| M_{i}^{2}$ the $i$-th message block composed of three chunks of 512 bits each $M_{i}^{j}$ and $S=\left[C_{0} C_{1} C_{2} C_{3}\right]$ the four 512-bit ECHO-columns constituting state $S$, we have $\left(H_{0}=I V\right)$ :

$$
C_{0} \leftarrow H_{i-1}, \quad C_{1} \leftarrow M_{i}^{0}, \quad C_{2} \leftarrow M_{i}^{1}, \quad C_{3} \leftarrow M_{i}^{2} .
$$

AES. We recall that one round, among the ten ones, of the AES-128 permutation is the succession of four transformations: SubBytes (SB), ShiftRows (SR), MixColumns (MC) and AddRoundKey (AK). We refer to the original publication [15] for further details.

Notations. We consider each state in the ECHO internal permutation, namely after each elementary transformations. We start with $S_{0}$, where the IV and the message are combined and end the first round after eight transformations in $S_{8}$. To refer to the AES-state at row $i$ and column $j$ of a particular ECHO-state $S_{n}$, we use the notation $S_{n}[i, j]$. Additionally, we introduce column-slice to refer to a thin column of size $16 \times 1$ of the ECHO state. The process of merging two lists $L_{1}$ and $L_{2}$ into a new list $L$ is denoted $L=L_{1} \bowtie L_{2}$. In the event that the merging should be done under some relation $t$, we use the operator $\bowtie_{|t|}$, where $|t|$ represents the size of the constraint to be verified in bits. Finally, in an AESstate, we consider four diagonals (from 0 to 3 ): diagonal $j \in[0,3]$ will be the four elements $(i, i+j(\bmod 4))$, with $i \in[0,3]$.

\subsection{Alternative Description}

For an easier description of some of the following attacks, we use an equivalent description of one round of the ECHO permutation. First, we swap the BigShiftRows transformation with the MixColumns transformation of the second AES round. Second, we swap SubBytes with ShiftRows of the first AES round. Swapping 
these operations does not change the computational result of ECHO and similar alternative descriptions have already been used in the analysis of AES. Hence, one round of ECHO results in the two transformations SuperSBox (SB-MC-SB) and SuperMixColumns (MC-BMC), which are separated just by byte-shuffling operation. The SuperSBox has first been analyzed by Daemen and Rijmen in [2] to study two rounds of AES and has been independently used by Lamberger et al. in [6] and Gilbert and Peyrin in [12] to analyze AES-based hash functions. The SuperMixColumns has been first introduced by Schläffer in [14] and reused in [4]. We refer to those articles for further details as well.

\section{Attack on the 5-Round ECHO-256 Hash Function}

In this section, we use a sparse truncated differential path and the properties of SuperMixColumns to get a collision attack on 5 rounds of the ECHO-256 hash function. The resulting complexity is $2^{112}$ with memory requirements of $2^{85.3}$. We first describe the truncated differential path (a truncated differential path only considers whether a byte of the state is active or not) and show how to find conforming input pairs. Due to the sparse truncated differential path, we are able to apply a rebound attack with multiple inbound phases to ECHO. Since at most one fourth of each ECHO state is active, we have enough freedom for two inbound phases and are also able to fully control the chaining input of the hash function.

\subsection{The Truncated Differential Path}

In the attack, we use two message blocks where the first block does not contain differences. For the second message block, we use the truncated differential path given in Figure 2. We use colors (red, yellow, green, blue, cyan) to describe different phases of the attack and to denote their resulting solutions. Active bytes are denoted by black color, and active AES states contain at least one active byte. Hence, the sequence of active AES states for each round of ECHO is as follows:

$$
5 \stackrel{r_{1}}{\longrightarrow} 16 \stackrel{r_{2}}{\longrightarrow} 4 \stackrel{r_{3}}{\longrightarrow} 1 \stackrel{r_{4}}{\longrightarrow} 4 \stackrel{r_{5}}{\longrightarrow} 16 .
$$

Note that in this path, we keep the number of active bytes low, except for the beginning and end. Therefore, we have enough freedom to find many solutions. We do not allow differences in the chaining input (blue) and in the padding (cyan). The last 16 bytes of the padding contain the message length and the two bytes above contain size of the hash function output. Note that the AES states containing the chaining values (blue) and padding (cyan) do not get mixed with other AES states until the first BigMixColumns transformation. Since the lower half of the state (row 2 and 3 ) is truncated to compute the final hash value, we force all differences to be in the lower half of the message: the feed-forward will then preserve that property. 

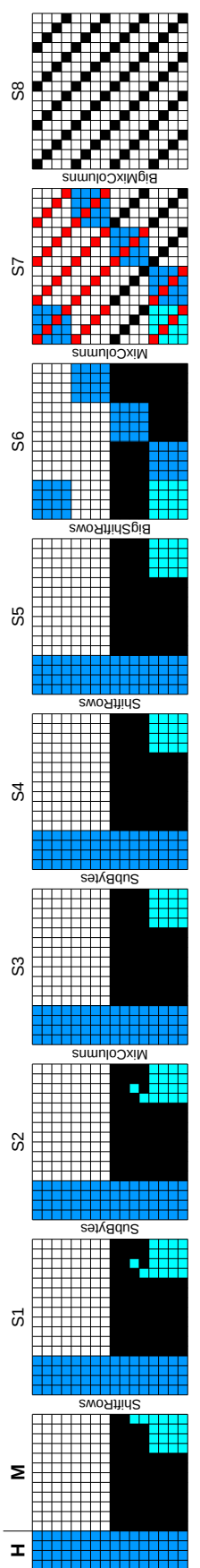

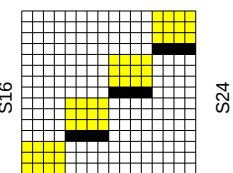

ПППППО
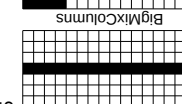

吅
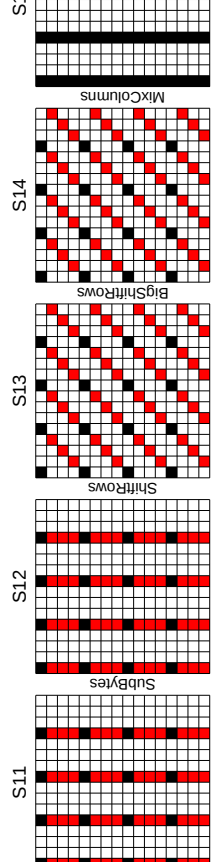

(1)
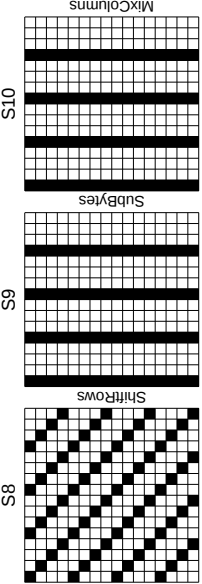
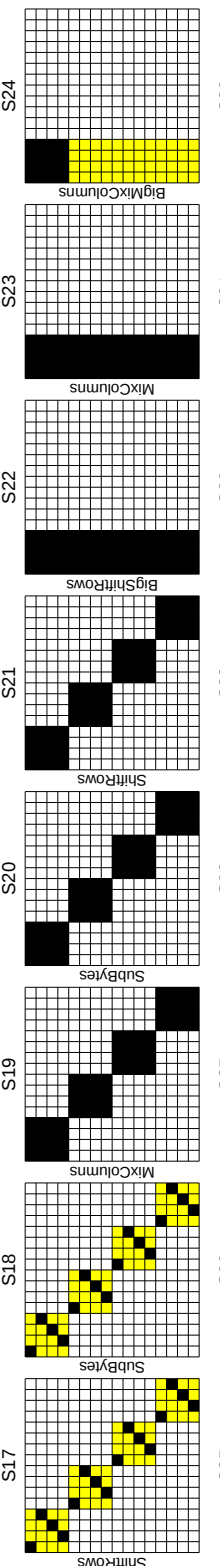

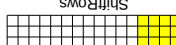
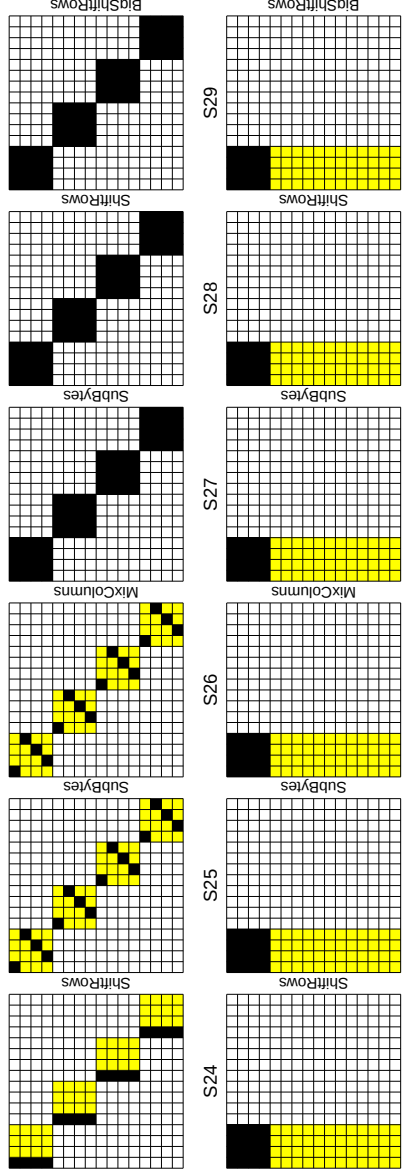
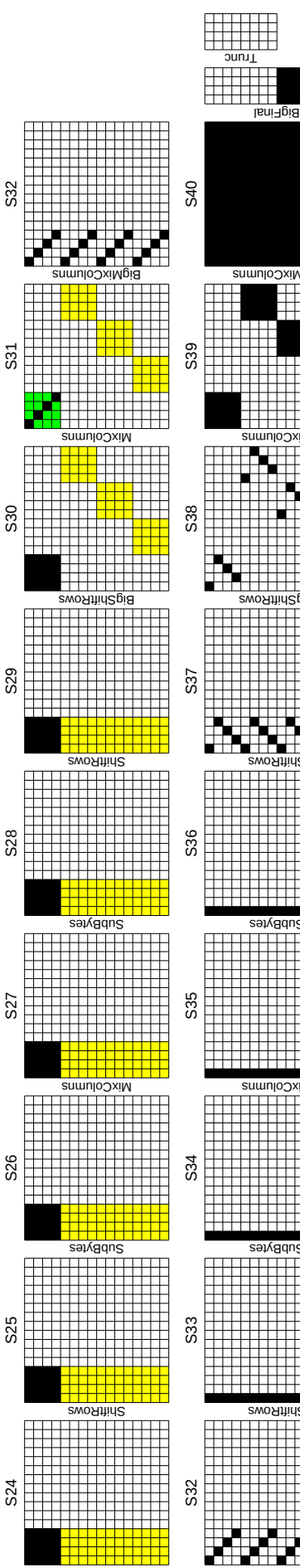

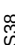

$\hat{\tilde{n}}$

$\stackrel{\circ}{\check{D}}$

范
خี

कै

ลิ

สี

ड.

تृ

$\cong$

그응

() $>$

.00

ฮี

胥

( )

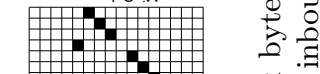

预

๑ 0

००

กิ .

虽

讯

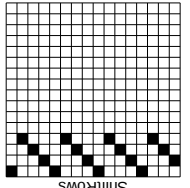

sмoун!!s

+ + (ำ

$10>$

రิ

శี ప્ర

?

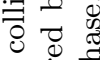

品 | | | | |

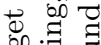

$\circ$ :

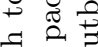

त्ञ

อ ฮี

疋

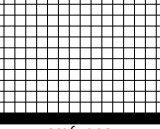

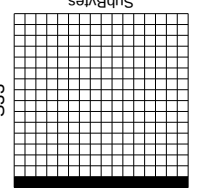

व

屯 200

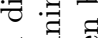

ชู ฮี่

त्व

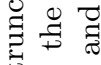

\#moynus

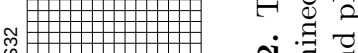

ง

so

我茎 


\subsection{Colliding Subspace Differences}

In the following, we show that the resulting output differences after 5 rounds lie in a vector space of reduced dimension. This can be used to construct a distinguisher for 5 rounds of the ECHO-256 hash function. However, due to the low dimension of the output vector space, we can even extend this subspace distinguisher to get a collision attack on 5 rounds of the ECHO-256 hash function.

First, we need to determine the dimension of the vector space at the output of the hash function. In general, the dimension of the output vector space is defined by the number of active bytes prior to the linear transformations in the last round (16 active bytes after the last SubBytes), combined with the number of active bytes at the input due to the feed-forward ( 0 active bytes in our case). This would results in a vector space dimension of $(16+0) \times 8=128$. However, a weakness in the combined transformations SuperMixColumns, BigFinal and the output truncation reduces the vector space to a dimension of 64 at the output of the hash function for the truncated differential path in Figure 2 .

We can move the BigFinal function prior to SuperMixColumns, since BigFinal is a linear transformation and the same linear transformation $\mathbf{M}_{\mathbf{S M C}}$ is applied to all columns in SuperMixColumns. Then, we get 4 active bytes at the same position in each AES state of the 4 resulting column-slices. To each active column-slice $C_{16}$, we first apply the SuperMixColumns multiplication with $\mathbf{M}_{\mathbf{S M C}}$ and then, a matrix multiplication using $\mathbf{M}_{\text {trunc }}=\left[I_{8} \mid 0_{8}\right]$ which truncates the lower 8 rows. Since only 4 bytes are active in $C_{16}$, these transformations can be combined into a transformation using a reduced $4 \times 8$ matrix $\mathbf{M}_{\text {comb }}$ applied to the reduced input $C_{4}$, which contains only the 4 active bytes of $C_{16}$ :

$$
\mathbf{M}_{\text {trunc }} \cdot \mathbf{M}_{\mathrm{SMC}} \cdot C_{16}=\mathbf{M}_{\mathbf{c o m b}} \cdot C_{4},
$$

The multiplication with zero differences of $C_{16}$ removes 12 columns of $\mathbf{M}_{\mathbf{S M C}}$ while the truncation removes 8 rows of $\mathbf{M}_{\mathbf{S M C}}$. For example, considering the first active column-slice leads to:

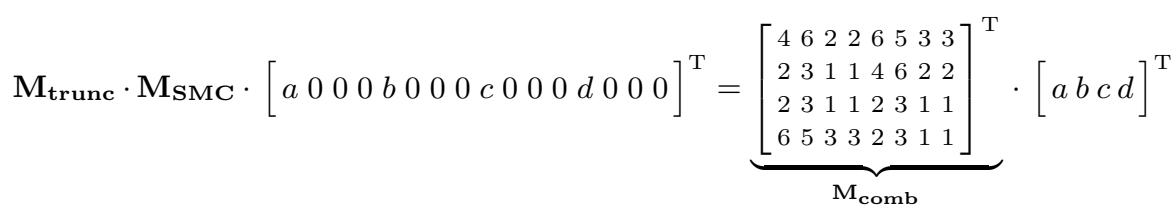

Analyzing the resulting matrix $\mathbf{M}_{\text {comb }}$ for all four active column-slices shows that in each case, the rank of $\mathbf{M}_{\text {comb }}$ is two, and not four. This reduces the dimension of the vector space in each active column-slice from 32 to 16. Since we have four active columns, the total dimension of the vector space at the output of the hash function is 64 . Furthermore, column $i \in\{0,1,2,3\}$ of the output hash value depends only on columns $4 i$ of state $S_{38}$. It follows that the output difference in the first column $i=0$ of the output hash value depends only on the four active differences in columns $0,4,8$ and 12 of state $S_{38}$, which we denote by $a$, $b, c$ and $d$. To get a collision in the first column of the hash function output, we get the following linear system of equations: 


$$
\mathbf{M}_{\mathbf{c o m b}} \cdot\left[\begin{array}{llll}
a & b & c & d
\end{array}\right]^{\mathrm{T}}=\left[\begin{array}{llllllll}
0 & 0 & 0 & 0 & 0 & 0 & 0 & 0
\end{array}\right]^{\mathrm{T}} .
$$

Since we cannot control the differences $a, b, c$ and $d$ in the following attack, we need to find a solution for this system of equations by brute-force. However, the brute-force complexity is less than expected due to the reduced rank of the given matrix. Since the rank is two, $2^{16}$ solutions exist and a random difference results in a collision with a probability of $2^{-16}$ instead of $2^{-32}$ for the first output column. Since the rank of all four output column matrices is two, we get a collision at the output of the hash function with a probability of $2^{-16 \times 4}=2^{-64}$ for the given truncated differential path.

\subsection{High-Level Outline of the Attack}

To find input pairs according to the truncated differential path given in Figure2, we use a rebound attack [9] with multiple inbound phases [6, [8]. The main advantage of multiple inbound phases is that we can first find pairs for each inbound phase independently and then, connect (or merge) the results. Furthermore, we also use multiple outbound phases and separate the merging process into three different parts which can be solved mostly independently:

1. First Inbound between $S_{16}$ and $S_{24}$ : find $2^{96}$ partial pairs (yellow and black bytes) with a complexity of $2^{96}$ in time and $2^{64}$ memory.

2. First Outbound between $S_{24}$ and $S_{31}$ : filter the previous solutions to get 1 partial pair (green, yellow and black bytes) with a complexity of $2^{96}$ in time and $2^{64}$ memory.

3. Second Inbound between $S_{7}$ and $S_{14}$ : find $2^{32}$ partial pairs (red and black) for each of the first three BigColumns and $2^{64}$ partial pairs for the last BigColumn of state $S_{7}$ with a total complexity of $2^{64}$ in time and memory.

4. First Part in Merging the Inbound Phases: combine the $2^{160}$ solutions of the previous phases according to the 128-bit SuperMixColumns condition given in [4]. We get $2^{32}$ partial pairs (black, red, yellow and green bytes between state $S_{7}$ and $S_{31}$ ) with complexity $2^{96}$ in time and $2^{64}$ memory.

5. Merge Chaining Input: repeat from Step 1 for $2^{16}$ times to get $2^{48}$ solutions for the previous phases. Compute $2^{112}$ chaining values (blue) using $2^{112}$ random first message blocks. Merge these solutions according to the overlapping 20 bytes (red with blue/cyan) in state $S_{7}$ to get $2^{48} \times 2^{112} \times 2^{-160}=1$ partial pair with complexity $2^{112}$ in time and $2^{48}$ memory.

6. Second Part in Merging the Inbound Phases: find one partial solution for the first two columns of state $S_{7}$ according to the 128-bit condition at SuperMixColumns between $S_{14}$ and $S_{16}$ with complexity $2^{64}$ in time and memory.

7. Third Part in Merging the Inbound Phases: find one solution for all remaining bytes (last two columns of state $S_{7}$ ) by fulfilling the resulting 192bit condition using a generalized birthday attack with 4 lists. The complexity is $2^{64}$ in time and memory to find one solution, and $2^{85.3}$ in time and memory to find $2^{64}$ solutions [16]. 
8. Second Outbound Phase to Get Collisions: in a final outbound phase, the resulting differences at the output of the hash function collide with a probability of $2^{-64}$ and we get one collision among the $2^{64}$ solutions of the previous step.

The total time complexity of the attack is $2^{112}$ and determined by Step 5; the memory complexity is $2^{85.3}$ and determined by Step 7 .

\subsection{Details of the Attack}

In this section, we describe the each phase of the collision attack on 5 rounds of ECHO-256 in detail. Note that some phases are also reused in the attacks on the compression function of Section 4 .

First Inbound between $S_{\mathbf{1 6}}$ and $S_{\mathbf{2 4}}$. We first search for internal state pairs conforming to the truncated differential path in round 3 (yellow and black bytes). We start the attack by choosing differences for the active bytes in state $S_{16}$ such that the truncated differential path of SuperMixColumns between state $S_{14}$ and $S_{16}$ is fulfilled (Section 2.1). We compute this difference forward to state $S_{17}$ through the linear layers.

We continue with randomly chosen differences of state $S_{24}$ and compute backwards to state $S_{20}$, the output of the SuperSBoxes. Since we have 64 active Sboxes in this state, the probability of a differential is about $2^{-1 \times 64}$. Hence, we need $2^{64}$ starting differences but get $2^{64}$ solutions for the inbound phase in round 3 (see 9$]$ ). We determine the right pairs for each of the 16 SuperSBox between state $S_{17}$ and $S_{20}$ independently. Using the Differential Distribution Table of the SuperSBoxes, we can find one right pair with average complexity one. In total, we compute $2^{96}$ solutions for this inbound phase with time complexity $2^{96}$ and memory complexity of at most $2^{64}$. For each of these pairs, differences and values of all yellow and black bytes in round 3 are determined.

Second Outbound between $S_{24}$ and $S_{31}$. In the outbound phase, we ensure the propagation in round 4 of the truncated differential path by propagating the right pairs of the previous inbound phase forwards to state $S_{31}$. With a probability of $2^{-96}$, we get four active bytes after MixColumns in state $S_{31}$ (green) conforming to the truncated path. Hence, among the $2^{96}$ right pairs of the inbound phase between $S_{16}$ and $S_{24}$ we expect to find one such right pair.

The total complexity to find this partial pair between $S_{16}$ and $S_{31}$ is then $2^{96}$. Note that for this pair, the values and differences of the yellow, green and black bytes between states $S_{16}$ and $S_{31}$ can be determined. Furthermore, note that for any choice of the remaining bytes, the truncated differential path between state $S_{31}$ and state $S_{40}$ is fulfilled.

Second Inbound between $S_{\mathbf{7}}$ and $\boldsymbol{S}_{\mathbf{1 4}}$. Here, we search for many pairs of internal states conforming to the truncated differential path between states $S_{7}$ and $S_{14}$. Note that we can independently search for pairs of each BigColumn 
of state $S_{7}$, since the four BigColumns stay independent until they are mixed by the following BigMixColumns transformation between states $S_{15}$ and $S_{16}$. For each BigColumn, four SuperSBoxes are active and we need at least $2^{16}$ starting differentials for each one to find the first right pair.

The difference in $S_{14}$ is already fixed due to the yellow inbound phase but we can still choose at least $2^{32}$ differences for each active AES state in $S_{7}$. Using the rebound technique, we can find one pair on average for each starting difference in the inbound phase. Then, we independently iterate through all $2^{32}$ starting differences for the first, second and third column and through all $2^{64}$ starting differences for the fourth column of state $S_{7}$. We get $2^{32}$ right pairs for each of the first three columns and $2^{64}$ pairs for the fourth column. The complexity to find all these pairs is $2^{64}$ in time and memory.

For each resulting right pair, the values and differences of the red and black bytes between states $S_{7}$ and $S_{14}$ can be computed. Furthermore, the truncated differential path in backward direction, except for two cyan bytes in the first states, is fulfilled. In the next phase, we partially merge the right pairs of the yellow and red inbound phase. But first, we recall the conditions for this merge.

First Part in Merging the Inbound Phases. For each pair of the previous two phases, the values of the red, yellow and black bytes of state $S_{14}$ and $S_{16}$ are fixed. These two states are separated by the linear SuperMixColumns transformation: taking the first column-slice as an example, we get

$$
\begin{array}{r}
\mathbf{M}_{\mathbf{S M C}} \cdot\left[\begin{array}{llllllllllllllll}
A_{0} & x_{0} & x_{1} & x_{2} & A_{1} & x_{3} & x_{4} & x_{5} & A_{2} & x_{6} & x_{7} & x_{8} & A_{3} & x_{9} & x_{10} & x_{11}
\end{array}\right]^{\mathrm{T}} \\
=\left[\begin{array}{llllllllllllllll}
B_{0} & B_{1} & B_{2} & B_{3} & y_{0} & y_{1} & y_{2} & y_{3} & y_{4} & y_{5} & y_{6} & y_{7} & y_{8} & y_{9} & y_{10} & y_{11}
\end{array}\right]^{\mathrm{T}},
\end{array}
$$

where $\mathbf{M}_{\mathbf{S M C}}$ is the SuperMixColumns transformation matrix, $A_{i}$ the input bytes determined by the red inbound phase and $B_{i}$ the output bytes determined by the yellow inbound phase. All bytes $x_{i}$ and $y_{i}$ are free to choose. As shown by Jean and Fouque [4], we only get a solution with probability $2^{-8}$ for each column-slice due to the low rank of the $\mathbf{M}_{\mathbf{S M C}}$ matrix. In [4] (Appendix A), the 8-bit condition for that particular column-slice that ensures the system to have solutions has been derived and is given as follows:

$$
2 \cdot A_{0}+3 \cdot A_{1}+A_{2}+A_{3}=14 \cdot B_{0}+11 \cdot B_{1}+13 \cdot B_{2}+9 \cdot B_{3} .
$$

Similar 8-bit conditions exist for all 16 column-slices. In total, each right pair of the two (independent) inbound phases results in a 128-bit condition on the whole SuperMixColumns transformation between states $S_{14}$ and $S_{16}$.

Remember that we have constructed one pair for the yellow inbound phase and in total, $2^{32} \times 2^{32} \times 2^{32} \times 2^{64}=2^{160}$ pairs for the red inbound phase. Among these $2^{160}$ pairs, we expect to find $2^{32}$ right pairs which also satisfy the 128 -bit condition of the SuperMixColumns between states $S_{14}$ and $S_{16}$. In the following, we show how to find all these $2^{32}$ pairs with a complexity of $2^{96}$.

First, we combine the $2^{32} \times 2^{32}=2^{64}$ pairs determined by the two first BigColumns of state $S_{7}$ in a list $L_{1}$ and the $2^{32} \times 2^{64}=2^{96}$ pairs determined by 
the last two BigColumns of state $S_{7}$ in a list $L_{2}$. Note that the pairs in these two lists are independent. Then, we separate Equation (11) into terms determined by $L_{1}$ and terms determined by $L_{2}$ :

$$
2 \cdot A_{0}+3 \cdot A_{1}=A_{2}+A_{3}+14 \cdot B_{0}+11 \cdot B_{1}+13 \cdot B_{2}+9 \cdot B_{3} .
$$

We apply the left-hand side to the elements of $L_{1}$ and the right-hand side to elements of $L_{2}$ and sort $L_{1}$ according to the bytes to be matched.

Then, we can simply merge (join) these lists to find those pairs which satisfy the 128-bit condition imposed by the SuperMixColumns and store these results in list $L_{12}=L_{1} \bowtie_{128} L_{2}$. This way, we get $2^{64} \times 2^{96} \times 2^{-128}=2^{32}$ right pairs with a total complexity of $2^{96}$. We note that the memory requirements can be reduced to $2^{64}$ if we do not store the elements of $L_{2}$ but compute them online. The resulting $2^{32}$ solutions are partial right pairs for the black, red, yellow and green bytes between state $S_{7}$ and $S_{31}$.

Merge Chaining Input. Next, we need to merge the $2^{32}$ results of the previous phases with the chaining input (blue) and the bytes fixed by the padding (cyan). The chaining input and padding overlap with the red inbound phase in state $S_{7}$ on $5 \times 4=20$ bytes. This results in a 160-bit condition on the overlapping blue/cyan/red bytes. To find a pair verifying this condition, we first generate $2^{112}$ random first message blocks, compute the blue bytes of state $S_{7}$ and store the results in a list $L_{3}$.

Additionally, we repeat $2^{16}$ times from the yellow inbound phase but with other starting point: 11 in state $S_{24}$. This way, we get $2^{16} \times 2^{32}=2^{48}$ right pairs for the combined yellow and red inbound phases, which also satisfy the 128-bit condition of SuperMixColumns between states $S_{14}$ and $S_{16}$. The complexity is $2^{16} \times 2^{96}=2^{112}$. We store the resulting $2^{48}$ pairs in list $L_{12}$.

Next, we merge the lists according to the overlapping 160-bits $\left(L_{12} \bowtie_{160} L_{3}\right)$ and get $2^{48} \times 2^{112} \times 2^{-160}=1$ right pair. If we compute the $2^{112}$ message blocks of list $L_{3}$ online, the time complexity of this merging step is $2^{112}$ with memory requirements of $2^{48}$. For the resulting pair, all differences between states $S_{4}$ and $S_{33}$ and all colored byte values (blue, cyan, red, yellow, green and black) between states $S_{0}$ and $S_{31}$ can be determined.

Second Part in Merging Inbound Phases. To completely merge the two inbound phases, we need to find according values for the white bytes. We use Figure 3 to illustrate the second and third part of the merge inbound phase. In this figure, we only consider values and therefore, do not show active bytes (black). Furthermore, all brown and cyan bytes have already been chosen in one of the previous steps. In the second part of the merge inbound phase, we only choose values for the gray and light-gray bytes. All other colored bytes show steps of the following merging phase.

We first choose random values for all remaining bytes of the two first columns in state $S_{7}$ (gray and light-gray) and independently compute the columns forward

${ }^{1}$ Until now, we have chosen only $2^{96}$ out of $2^{128}$ differences for this state. 


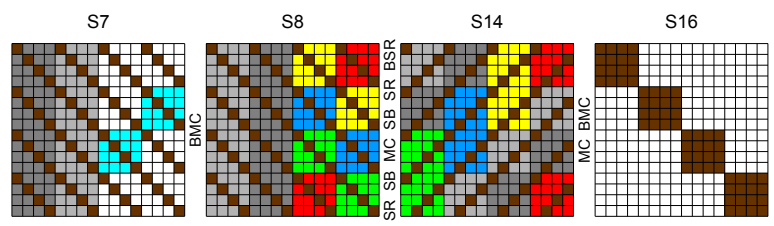

Fig. 3. States used to merge the two inbound phases with the chaining values. The merge inbound phase consists of three parts. Brown bytes show values already determined (first part) and gray values are chosen at random (second part). Green, blue, yellow and red bytes show independent values used in the generalized birthday attack (third part) and cyan bytes represent values with the target conditions.

to state $S_{14}$. Note that we need to try $2^{2 \times 8+1}$ values for AES state $S_{7}[2,1]$ to also match the 2-byte (cyan) and 1-bit padding at the input in AES state $S_{0}[2,3]$. Then, all gray, light-gray, cyan and brown bytes have already been determined either by an inbound phase, chaining value, padding or just by choosing random values for the remaining free bytes of the two first columns of $S_{7}$. However, all white, red, green, yellow and blue bytes are still free to choose.

By considering the linear SuperMixColumns transformation, we observe that in each column-slice, 14 out of 32 input/output bytes are already fixed and 2 bytes are still free to choose. Hence, we expect to get $2^{16}$ solutions for this linear system of equations. Unfortunately, also for the given position of already determined 14 bytes, the linear system of equations does not have a full rank. Again, we can determine the resulting system using the matrix $\mathbf{M}_{\mathbf{S M C}}$ of SuperMixColumns. As an example, for the first column-slice, the system is given as follows:

$$
\begin{aligned}
& \mathbf{M}_{\mathbf{S M C}} \cdot\left[\begin{array}{llllllllllllllll}
A_{0} & L_{0} & L_{1} & L_{2} & A_{1} & L_{0}^{\prime} & L_{1}^{\prime} & L_{2}^{\prime} & A_{2} & x_{6} & x_{7} & x_{8} & A_{3} & x_{9} & x_{10} & x_{11}
\end{array}\right]^{\mathrm{T}} \\
& =\left[\begin{array}{llllllllllllllll}
B_{0} & B_{1} & B_{2} & B_{3} & y_{0} & y_{1} & y_{2} & y_{3} & y_{4} & y_{5} & y_{6} & y_{7} & y_{8} & y_{9} & y_{10} & y_{11}
\end{array}\right]^{\mathrm{T}} \text {. }
\end{aligned}
$$

The free variables in this system are $x_{6}, \ldots, x_{11}$ (green). The values $A_{0}, A_{1}$, $A_{2}, A_{3}, B_{0}, B_{1}, B_{2}, B_{3}$ (brown) have been determined by the first or second inbound phase and the values $L_{0}, L_{1}, L_{2}$ (light-gray) and $L_{0}^{\prime}, L_{1}^{\prime}, L_{2}^{\prime}$ (gray) are determined by the choice of arbitrary values in state $S_{7}$. We proceed as before and determine the linear system of equations which needs to have a solution:

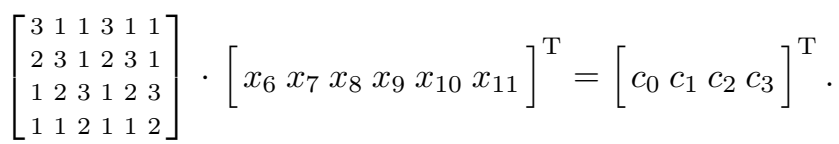

The resulting linear 8-bit equation to get a solution for this system can be separated into terms depending on values of $L_{i}$ and on $L_{i}^{\prime}$, and we get $f_{1}\left(L_{i}\right)+$ $f_{2}\left(L_{i}^{\prime}\right)+f_{3}\left(a_{i}, b_{i}\right)=0$, where $f_{1}, f_{2}$ and $f_{3}$ are linear functions. For all other 16 column-slices and fixed positions of gray bytes, we get matrices of rank three as well. In total, we get 16 8-bit conditions and the probability to find a solution for a given choice of gray and light-gray values in states $S_{14}$ and $S_{16}$ is $2^{-128}$. 
However, we can find a solution to these linear equations using the birthday effect and a meet-in-the-middle attack with a complexity of $2^{64}$ in time and memory.

We start by choosing $2^{64}$ values for each of the first (gray) and second (lightgray) BigColumns in state $S_{7}$. We compute these values independently forward to state $S_{14}$ and store them in two lists $L$ and $L^{\prime}$. We also separate all equations of the 128-bit condition into parts depending only on values of $L$ and $L^{\prime}$. We apply the resulting functions $f_{1}, f_{2}, f_{3}$ to the elements of lists $L_{i}$ and $L_{i}^{\prime}$, and merge two lists $L \bowtie_{128} L^{\prime}$ using the birthday effect.

Third Part in Merging Inbound Phases. We continue with a generalized birthday match to find values for all remaining bytes of the state (blue, red, green, yellow, cyan and white of Figure 3). For each column in state $S_{14}$, we independently choose $2^{64}$ values for the green, blue, yellow and red columns, and compute them independently backward to $S_{8}$. We need to match the values of the cyan bytes of state $S_{7}$, which results in a condition on 24 bytes or 192 bits. Since we have four independent lists with $2^{64}$ values in state $S_{8}$, we can use the generalized birthday attack [16] to find one solution with a complexity of $2^{192 / 3}=2^{64}$ in time and memory.

In more detail, we need to match values after the BigMixColumns transformation in the backward direction. Hence, we first multiply each byte of the four independent lists by the four multipliers of the InvMixColumns transformation. Then, we get 24 equations containing only XOR conditions on bytes between the target value and elements of the four independent lists, which can be solved using a generalized birthday attack.

To improve the average complexity of this generalized birthday attack, we can start with larger lists for the green, blue, yellow and red columns in state $S_{14}$. Since we need to match a 192 -bit condition, we can get $2^{3 \cdot x} \times 2^{-192}=2^{x}$ solutions with a time and memory complexity of $\max \left\{2^{64}, 2^{x}\right\}$ (see [16] for more details). Note that we can even find solutions with an average complexity of 1 using lists of size $2^{96}$. Each solutions of the generalized birthday match results in a valid pair conforming to the whole 5-round truncated differential path.

Second Outbound Phase to Get Collisions. For the collision attack on 5 rounds, we start the generalized birthday attack of the previous phase with lists of size $2^{85.3}$. This results in $2^{3 \cdot 85.3} \times 2^{-192}=2^{64}$ solutions with a time and memory complexity of $2^{85.3}$, or with an average complexity of $2^{21.3}$ per solution. These solutions are propagated outwards in a second, independent outbound phase. Since the differences at the output collide with a probability of $2^{-64}$, we expect to find one pair which collides at the output of the hash function. The time complexity is determined by merging the chaining input and the memory requirements by the generalized birthday attack. To summarize, the complexity to find a collision for 5 rounds of the ECHO-256 hash function is given by about $2^{112}$ compression function evaluations with memory requirements of $2^{85.3}$. 


\section{Distinguisher on the 7-Round ECHO-256 Compression Function}

In this section, we detail our distinguisher on 7 rounds in the known-salt model. First, we show how to obtain partial solutions that verify the path from the state $S_{6}$ to $S_{23}$ with an average complexity of $2^{64}$ in time, as we obtain $2^{64}$ solutions with a cost of $2^{128}$. These partial solutions determine also the values of the blue bytes (in Figure 4). Next, we show how to do the same for the yellow part of the path from $S_{30}$ to $S_{47}$. Finally, we explain how to merge these partial solutions for finding one that verifies the whole path.

\subsection{Finding Pairs between $S_{6}$ and $S_{23}$}

We explain here how to find $2^{64}$ solutions for the blue part with a cost of $2^{128}$ in time and $2^{64}$ in memory. This is done with a stop-in-the-middle algorithm similar to the one presented in [11] for improving the time complexity of the ECHO-256 distinguisher. This algorithm has to be adapted to this particular situation, where all the active states belong to the same BigColumn.

We start by fixing the difference in $S_{8}$ to a chosen value, so that the transition between $S_{6}$ and $S_{8}$ is verified. We fix the difference in the active diagonals of the two AES-states $S_{23}[0,0]$ and $S_{23}[3,1]$ to a chosen value.

From state $S_{8}$ to $S_{13}$, we have four different SuperSBox groups involved in the active part. From states $S_{16}$ to $S_{22}$, we have $4 \times 4$ SuperSBox groups involved (4 per active AES state). Those 16 groups, as well as the 4 previous ones, are completely independent from $S_{16}$ to $S_{22}$ (respectively from $S_{8}$ to $S_{13}$ ). From the known difference in $S_{8}$, we build four lists of values and differences in $S_{13}$ : each list corresponds to one of the four SuperSBox groups. Each list is of size $2^{32}$ because once we know the input difference, we try all the possible $2^{32}$ possible values and then we can compute the values and differences in $S_{13}$ (as we said, the four groups are independent in this part of the path). In the sequel, those lists are denoted $L_{A}^{0}, L_{A}^{1}, L_{A}^{2}$ and $L_{A}^{3}$.

There are 64 bits of differences not yet fixed in $S_{23}$. Each active diagonal only affects the AES state where it is in, so we can independently consider $2^{32}$ possible differences for one diagonal and $2^{32}$ differences for the other. We can now build the 16 lists corresponding to the 16 SuperSBox groups as we did before, but considering that: the 8 lists corresponding to 8 groups of the two AES states $S_{16}[0,0]$ and $S_{16}[3,0]$, as they have their differences in $S_{22}$ already fixed, have a size of $2^{32}$ (corresponding to the possible values for each group). These are the lists $L_{0,0}^{i}$ and $L_{3,0}^{i}$, with $i \in[0,3]$ that represents the $i$ th diagonal of the state. But the lists $L_{1,0}^{i}, L_{2,0}^{i}$, with $i \in[0,3]$, as they do not have yet the difference fixed, have a size of $2^{32+32}$ each, as we can consider the $2^{32}$ possible differences for each not fixed diagonal independently. Next, we go through the $2^{64}$ possible differences of the two first diagonals (diagonals 0 and 1) of the active AES state in $S_{15}$. For each one of these $2^{64}$ possible differences: 


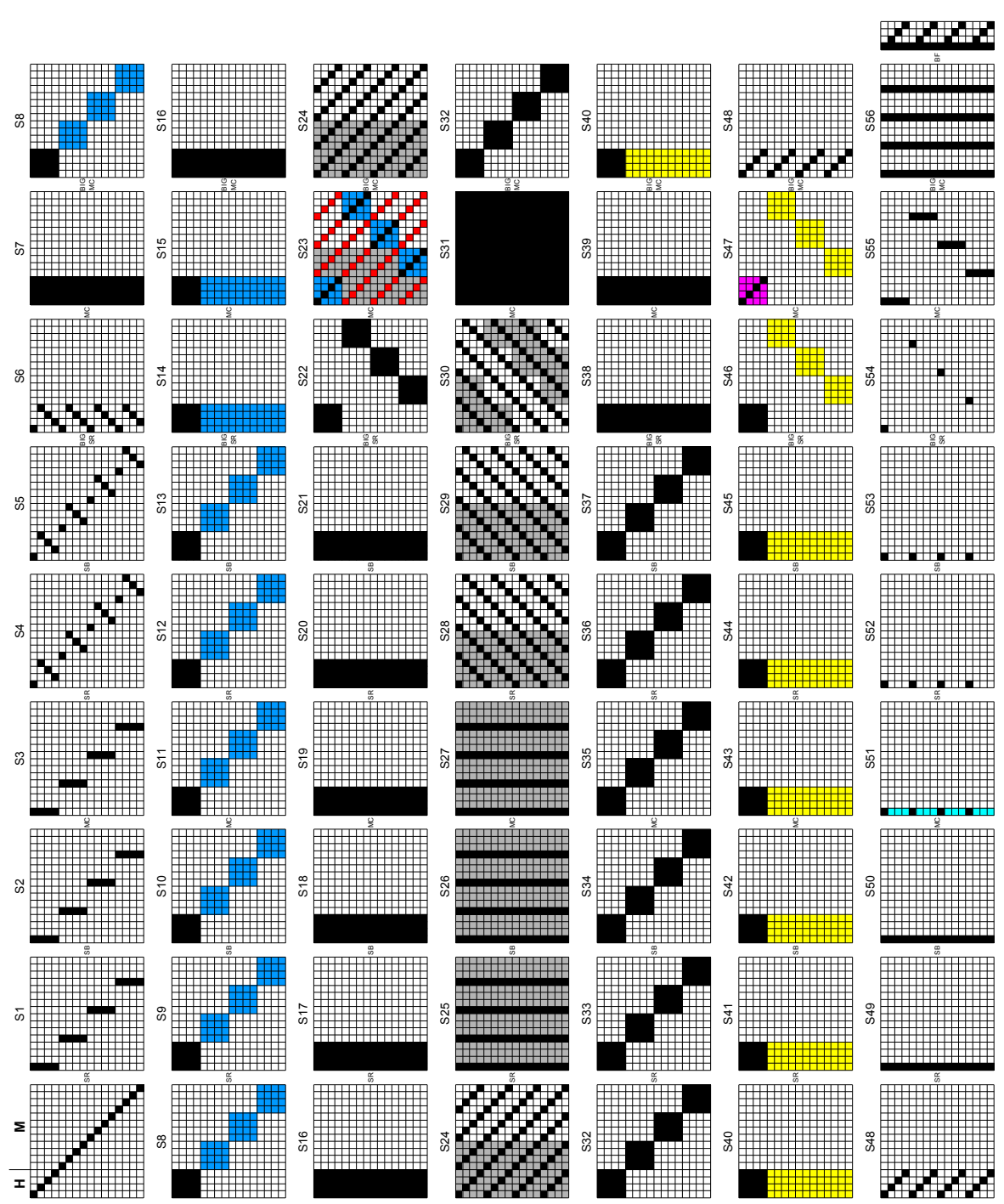

Fig. 4. Differential path for the seven-round distinguisher

- The associated differences in the two same diagonals in the four active AES states of $S_{16}$ can be computed. Consequently, we can check in the previously computed ordered lists $L_{j, 0}^{i}$ with $j \in[0,3]$ and $i \in[0,1]$ where we find this difference 2 . For $j \in\{0,3\}$, on average, we obtain one match on each one of the lists $L_{0,0}^{0}, L_{0,0}^{1}, L_{3,0}^{0}$ and $L_{3,0}^{1}$. For $j \in\{1,2\}$, we obtain $2^{32}$ matches, one for each of the $2^{32}$ possible differences in the associated diagonals in $S_{23}$. That is $2^{32}$ matches for $L_{1,0}^{0}$ and $L_{1,0}^{1}$, where a pair of values formed

$\overline{{ }^{2} i \text { is either } 0}$ or 1 because we are just considering the two first diagonals. 
by one element of each list is only valid if they were generated from the same difference in $S_{23}$. Consequently, we can construct the list $L_{1,0}^{0,1}$ of size $2^{32}$ where we store the values and differences of those two diagonals in the AES state $S_{16}[1,0]$ as well as the difference in $S_{23}$ from which they were generated. Repeating the process for $L_{2,0}^{0}$ and $L_{2,0}^{1}$, we construct the list $L_{2,0}^{0,1}$ of size $2^{32}$. We can merge the lists $L_{1,0}^{0,1}, L_{2,0}^{0,1}$ and the four fixed values for differences and values obtained from the matches in the lists $L_{0,0}^{0}, L_{0,0}^{1}$, $L_{3,0}^{0}$ and $L_{3,0}^{1}$, corresponding to the AES states $S_{16}[0,0]$ and $S_{16}[3,0]$. This generates the list $L^{0,1}$ of size $2^{64}$. Each element of this list contains the values and differences of the two diagonals 0 and 1 of the four active AES states in $S_{16}$. As we have all the values for the two first diagonals in the four AES states, for each one of these elements, we compute the values in the two first diagonals of the active state in $S_{15}$ by applying the inverse of BigMixColumns. We order them according to these values.

- Next, we go through the $2^{64}$ possible differences of the two next diagonals (diagonals 2 and 3 ) of the active AES state in $S_{15}$. For each one of these $2^{64}$ possible differences:

- All the differences in the AES state $S_{13}[0,0]$ are determined. We check in the lists $L_{A}^{0}, L_{A}^{1}, L_{A}^{2}$ and $L_{A}^{3}$ if we find a match for the differences. We expect to find one in each list and this determines the values for the whole state $S_{15}[0,0]$ (as the elements in these lists are formed by differences and values). This means that the value of the active AES state in $S_{15}$ is also completely determined. This way, we can check in the previously generated list $L^{0,1}$ if the correct value for the two diagonals 0 and 1 appears. We expect to find it once.

- As we have just found a valid element from $L^{0,1}$, it determines the differences in the AES states $S_{23}[1,0]$ and $S_{23}[2,0]$ that were not fixed yet. Now, we need to check if, for those differences in $S_{23}$, the corresponding elements in the four lists $L_{1,0}^{i}, L_{2,0}^{i}$ for $i \in[2,3]$ that match with the differences fixed in the diagonals 2 and 3 of $S_{15} \sqrt{3}$, satisfy the values in $S_{15}$ that were also determined by the lists $L_{A}^{i}$. This occurs with probability $2^{-64}$.

All in all, the time complexity of this algorithm is $2^{64} \cdot\left(2^{64}+2^{64}\right)=2^{129}$ with a memory requirement of $2^{64}$. The resulting expected number of valid pairs is $2^{64} \cdot 2^{64} \cdot 2^{64} \cdot 2^{-64} \cdot 2^{-64}=2^{64}$.

\subsection{Finding Pairs between $S_{30}$ and $S_{47}$}

In quite the same way as the previous section, we can find solutions for the yellow part with an average cost of $2^{64}$. To do so, we take into account the fact that the MixColumns and BigMixColumns transformations commute. So, if we exchange their positions between states $S_{39}$ and $S_{40}$, we only have one active AES state in $S_{39}$. We fix the differences in $S_{47}$ and in two AES states, say $S_{32}[0,0]$ and

\footnotetext{
${ }^{3}$ We expect one match per list.
} 
$S_{32}[1,1]$, and we still have $2^{32}$ possible differences for each of the two remaining active AES states in $S_{32}$. Then, the lists $L_{A}^{i}$ are generated from the end and contain values and differences from $S_{40}$. Similarly, the lists $L_{j, j}^{i}$ contain values and differences from $S_{38}$. We can apply the same algorithm as before and obtain $2^{64}$ solutions with a cost of $2^{128}$ in time and $2^{64}$ in memory.

\subsection{Merging Solutions}

In this section, we explain how to get a solution for the whole path. As explained in our Section 4.1, we can find $2^{64}$ solutions for the blue part, that have the same difference for the active AES states of columns 0 and 1 in $S_{23}$. We obtain $2^{64}$ solutions from a fixed value for the differences in $S_{8}$ and the AES states $S_{23}[0,0]$ and $S_{23}[3,1]$. Repeating this process for the $2^{32}$ possible differences in $S_{8}$, we obtain in total $2^{96}$ solutions for the blue part with the same differences in the columns 0 and 1 in $S_{23}$. The cost of this step is $2^{160}$ in time and $2^{96}$ in memory.

The same way, using the algorithm explained in Section 4.2, we can also find $2^{96}$ solutions for the yellow part, that have the same difference value for the AES active states of columns 0 and 1 in $S_{32}$ (we fix the difference value of this two columns in $S_{32}$, and we try all the $2^{32}$ possible values for the difference in $S_{47}$ ). The cost of this step is also $2^{160}$ in time and $2^{96}$ in memory.

Now, from the partial solutions obtained in the previous steps, we want to find a solution that verifies the whole differential path. For this, we want to merge the solutions from $S_{23}$ with the solutions from $S_{32}$. We know that the differences of the columns 0,1 of $S_{24}$ and $S_{31}$ are fixed. Hence, from $S_{24}$ to $S_{31}$, there are four AES states for which we know the input difference and the output difference, as they are fixed. We can then apply a variant of the SuperSBox [3, 6] technique in these four AES states: it fixes the possible values for the active diagonals of those states.

The differences in the other four AES states in $S_{24}$ that are fixed are associated to other differences that are not fixed 5 . There are $2^{64}$ possible differences, each one associated to $2^{32}$ solutions for $S_{32}-S_{47}$ given by the solutions that we found in the second step. For each one of these $2^{64}$ possible differences, one possible value is associated by the SuperSBox. When computing backwards these values to state $S_{24}$, as we have also the values for the other four AES states of the columns 0 and 1 that are also fixed (in the third step), we can compute the values for these two columns in $S_{23}$, and we need $32 \times 2$ bit conditions to be verified on the values. So for each one of the $2^{64}$ possible differences in $S_{31}$, we obtain $2^{96-64}=2^{32}$ that verify the conditions on $S_{23}$. In total, we have $2^{64+32}=2^{96}$ possible partial matches.

For each of the $2^{64}$ possible differences in $S_{31}$, its associated $2^{32}$ possible partial matches also need to verify the 128-bit condition in $S_{30}-S_{32}$ at the SuperMixColumns layer [4] and the remaining $2 \times 32$ bit conditions on the values

\footnotetext{
${ }^{4} S_{24}[0,0], S_{24}[0,1], S_{24}[1,1], S_{24}[3,0]$ correspond to $S_{31}[0,0], S_{31}[0,1], S_{31}[1,0]$, $S_{31}[3,1]$, respectively.

${ }^{5} S_{24}[1,0], S_{24}[2,0], S_{24}[2,1], S_{24}[3,1]$ correspond to $S_{31}[1,3], S_{31}[2,2], S_{31}[2,3]$, $S_{31}[3,2]$.
} 
of $S_{23}$. Since for each of the $2^{64}$ differences we have $2^{32}$ possible associated values in $S_{32}$, the probability of finding a good pair is $2^{96-128-64+32}=2^{-64}$.

If we repeat this merging procedure $2^{64}$ times, namely for $2^{32}$ differences in the columns 0 and 1 of $S_{23}$ and for $2^{32}$ differences in the columns 0 and 1 of $S_{32}$, we should find a solution. We then repeat the procedure for the cross product of the $2^{32}$ solutions for each side. As we do not want to compute them each time that we use them, as it would increase the time complexity, we can just store the $2^{64+32+32}=2^{128}$ solutions for the first part and use the corresponding ones when needed, while the second part is computed in sequence. The complexity would be: $2^{192}+2^{192}+2^{96+64}$ in time and $2^{128}$ in memory. So far, we have found a partial solution for the differential part for rounds from $S_{6}$ to $S_{48}$. We still have the passive bytes to determine and the condition to pass from $S_{50}$ to $S_{51}$ to verify. This can be done exactly as in the second and third part of the merge inbound phase of Section 3.4 with no additional cost.

Moreover, since we can find $x$ solutions with complexity $\max \left\{x, 2^{96}\right\}$ in time and $2^{96}$ memory for the (independent) merge inbound phase, we can get $x<2^{193}$ solutions with time complexity $2^{193}+\max \left\{x, 2^{96}\right\} \sim 2^{193}$ and $2^{128}$ memory. We need only $2^{96}$ of these solutions to pass the probabilistic propagation in the last round from $S_{50}$ to $S_{51}$. Hence, we can find a complete solution for the whole path with a cost of about $2^{193}$ in time and $2^{128}$ in memory. Furthermore, with a probability of $2^{-128}$, the input and output differences in $S_{0}$ and $S_{48}$ collide in the feed-forward and BigFinal transformation. Therefore, we can also generate freestart collisions for 6 rounds of the compression function with a time complexity of $2^{193}+2^{128} \sim 2^{193}$ and $2^{128}$ memory.

\section{Conclusions}

In this work, we have presented new results on the second-round candidate of the SHA-3 competition ECHO-256 that improve considerably the previous published cryptanalysis. Our analysis are based on multi-inbound rebound attacks and are summarized in Table 11. The main results are a 5-round collision of the hash function and a 7-round distinguisher of its compression function. All of our results take into account the condition observed in [4], which is needed to merge the results of multiple inbound phases, and satisfy it. The 7-round distinguisher on the compression function uses the stop-in-the-middle algorithms proposed in [10].

\section{References}

1. Benadjila, R., Billet, O., Gilbert, H., Macario-Rat, G., Peyrin, T., Robshaw, M., Seurin, Y.: SHA-3 proposal: ECHO. Submission to NIST (updated) (2009), http://crypto.rd.francetelecom.com/echo/doc/ echo_description_1-5.pdf

2. Daemen, J., Rijmen, V.: Understanding Two-Round Differentials in AES. In: De Prisco, R., Yung, M. (eds.) SCN 2006. LNCS, vol. 4116, pp. 78-94. Springer, Heidelberg (2006) 
3. Gilbert, H., Peyrin, T.: Super-Sbox Cryptanalysis: Improved Attacks for AESLike Permutations. In: Hong, S., Iwata, T. (eds.) FSE 2010. LNCS, vol. 6147, pp. 365-383. Springer, Heidelberg (2010)

4. Jean, J., Fouque, P.-A.: Practical Near-Collisions and Collisions on Round-Reduced ECHO-256 Compression Function. In: Joux, A. (ed.) FSE 2011. LNCS, vol. 6733, pp. 107-127. Springer, Heidelberg (2011)

5. Jean, J., Naya-Plasencia, M., Schläffer, M.: Improved Analysis of ECHO-256. Cryptology ePrint Archive, Report 2011/422 (2011), http://eprint.iacr.org/

6. Lamberger, M., Mendel, F., Rechberger, C., Rijmen, V., Schläffer, M.: Rebound Distinguishers: Results on the Full Whirlpool Compression Function. In: Matsui, M. (ed.) ASIACRYPT 2009. LNCS, vol. 5912, pp. 126-143. Springer, Heidelberg (2009)

7. Van Le, T., Sparr, R., Wernsdorf, R., Desmedt, Y.G.: Complementation-Like and Cyclic Properties of AES Round Functions. In: Dobbertin, H., Rijmen, V., Sowa, A. (eds.) AES 2005. LNCS, vol. 3373, pp. 128-141. Springer, Heidelberg (2005)

8. Matusiewicz, K., Naya-Plasencia, M., Nikolić, I., Sasaki, Y., Schläffer, M.: Rebound Attack on the Full LANE Compression Function. In: Matsui, M. (ed.) ASIACRYPT 2009. LNCS, vol. 5912, pp. 106-125. Springer, Heidelberg (2009)

9. Mendel, F., Rechberger, C., Schläffer, M., Thomsen, S.S.: The Rebound Attack: Cryptanalysis of Reduced Whirlpool and Grøst1. In: Dunkelman, O. (ed.) FSE 2009. LNCS, vol. 5665, pp. 260-276. Springer, Heidelberg (2009)

10. Naya-Plasencia, M.: How to Improve Rebound Attacks. In: Rogaway, P. (ed.) CRYPTO 2011. LNCS, vol. 6841, pp. 188-205. Springer, Heidelberg (2011)

11. Naya-Plasencia, M.: How to Improve Rebound Attacks. Cryptology ePrint Archive, Report 2010/607 (2010) (extended version), http://eprint.iacr.org/

12. Peyrin, T.: Improved Differential Attacks for ECHO and Grøstl. In: Rabin, T. (ed.) CRYPTO 2010. LNCS, vol. 6223, pp. 370-392. Springer, Heidelberg (2010)

13. Sasaki, Y., Li, Y., Wang, L., Sakiyama, K., Ohta, K.: Non-Full-Active Super-Sbox Analysis: Applications to ECHO and Grøstl. In: Abe, M. (ed.) ASIACRYPT 2010. LNCS, vol. 6477, pp. 38-55. Springer, Heidelberg (2010)

14. Schläffer, M.: Subspace Distinguisher for 5/8 Rounds of the ECHO-256 Hash Function. In: Biryukov, A., Gong, G., Stinson, D.R. (eds.) SAC 2010. LNCS, vol. 6544, pp. 369-387. Springer, Heidelberg (2011)

15. National Institute of Standards, Technology (NIST): Advanced E.D.F.G.D.F.ncryption Standard (FIPS PUB 197) (November 2001), http://www.csrc.nist.gov/publications/fips/ fips197/fips-197.pdf

16. Wagner, D.: A Generalized Birthday Problem. In: Yung, M. (ed.) CRYPTO 2002. LNCS, vol. 2442, pp. 288-303. Springer, Heidelberg (2002) 